OPEN ACCESS

Edited by:

Paola Ulivi,

Scientific Institute of Romagna for the

Study and Treatment of Tumors

(IRCCS), Italy

Reviewed by:

Hua Wang,

Anhui Medical University, China

*Correspondence:

Xun $\mathrm{Li}$

154964740@qq.com

Shisan Bao

profbao@hotmail.com

Specialty section: This article was submitted to

Gastroenterology,

a section of the journal

Frontiers in Medicine

Received: 08 November 2021

Accepted: 10 January 2022

Published: 02 February 2022

Citation:

Dang J, He Z, Cui X, Fan J, Hambly DJ, Hambly BD, LiX and Bao S (2022) The Role of IL-37 and

IL-38 in Colorectal Cancer.

Front. Med. 9:811025.

doi: $10.3389 /$ fmed.2022.811025

\section{The Role of IL-37 and IL-38 in Colorectal Cancer}

\author{
Jie Dang ${ }^{1}$, Zhiyun $\mathrm{He}^{2}$, Xiang Cui ${ }^{3}$, Jingchun Fan ${ }^{4}$, David J. Hambly ${ }^{5}$, Brett D. Hambly ${ }^{4,6}$, \\ Xun $\mathrm{Li}^{2 *}$ and Shisan Bao ${ }^{4 *}$ \\ ${ }^{1}$ Child and Adolescent Health Management Center, Lanzhou University Second Hospital, Lanzhou, China, ${ }^{2}$ Department of \\ General Surgery, Lanzhou University First Hospital, Lanzhou, China, ${ }^{3}$ Department of General Surgery, Lanzhou University \\ Second Hospital, Lanzhou, China, ${ }^{4}$ Department of Epidemiology and Evidence-Based Medicine, School of Public Health, \\ Centre for Evidence-Based Medicine, Gansu University of Chinese Medicine, Lanzhou, China, ${ }^{5}$ Resident Training Program, \\ Gold Coast University Hospital, Southport, QLD, Australia, ${ }^{6}$ Centre for Healthy Futures, Torrens University Australia, Sydney, \\ NSW, Australia
}

Colorectal cancer $(\mathrm{CRC})$ is a major killer. Dysregulation of IL-37 and IL-38, both anti-inflammatory cytokines, is observed in auto-immune diseases. The precise regulatory mechanisms of IL-37/IL-38 during the development of CRC remains unclear, but chronic intestinal inflammation is involved in the carcinogenesis of CRC. Constitutive production of colonic IL-37 and IL-38 is substantially reduced in CRC, consistent with an inverse correlation with $\mathrm{CRC}$ differentiation. Reduced colonic IL-37 and IL-38 is relating to $\mathrm{CRC}$ invasion and distant metastasis, suggesting a protective role for IL-38 within the tumor micro-environment. IL-38 is reduced in right-sided CRC compared to left-sided $\mathrm{CRC}$, which is in line with multiple risk factors for right-sided $\mathrm{CRC}$, including the embryonic development of the colon, and genetic differences in CRC between these two sides. Finally, colonic IL-37 and tumor associated neutrophils (TAN) seem to be independent biomarkers of prognostic value, whereas colonic IL-38 seems to be a reliable and independent biomarker in predicting the 5-year survival post-surgery in CRC. However, there is room for improvement in available studies, including the extension of these studies to different regions/countries incorporating different races, evaluation of the role of multi-drug resistance, and different subsets of CRC. It would be useful to determine the kinetics of circulating IL-38 and its relationship with drug resistance/targeted therapy. The measurement of colonic IL-38 at the molecular and cellular level is required to explore the contribution of IL-38 pathways during the development of CRC. These approaches could provide insight for the development of personalized medicine.

Keywords: IL-37, IL-38, colorectal cancer, TAM, TAN

\section{IL-37}

IL-37 belongs to the IL-1 superfamily (1) that shares the structural pattern of the IL-1 family (2). IL-37 is a $17-26 \mathrm{kDa}$ protein that corresponds with a gene size of $3.617 \mathrm{~kb}$ (3). Constitutive expression of IL-37 has been observed in many leucocytes (NK cells, activated B cells, monocytes), epithelial cells (keratinocytes and epithelial cells), and tissues (e.g., lymph nodes, thymus, lung, intestine, and uterus) $(4,5)$. IL-37 is mainly considered to be an anti-inflammatory cytokine, because IL-37 is able to suppress innate (2) and adaptive immunity (6), thus explaining the 
consequent reduction of the host immune response (7), including in tumorigenesis (8). The anti-inflammatory function of IL37 (7) is partially due to the capacity of IL-37 to inhibit the maturation of dendritic cells (9), and/or to regulate polarization of macrophages, i.e., promoting M1 but inhibiting M2 macrophages (10). Dysregulated IL-37 has been reported in a number of auto-immune diseases, e.g., rheumatoid arthritis (11), psoriasis, Grave's disease and systemic lupus erythematous, and in ulcerative colitis and Crohn's disease (12), and in gestational diabetes mellitus (13), acting perhaps via inhibiting the expression and function of pro-inflammatory cytokines $(11,12,14)$. In addition, it has been well-reviewed that IL37 substantially reduces the development of atherosclerosis (15), which has been confirmed in an IL-37 transgenic animal model (16).

\section{IL-38}

IL-38 also belongs to the IL-1 superfamily, which shares $\sim 41 \%$ homology with the IL-1Ra and $\sim 43 \%$ homology with the IL-36R (17). IL-38 is constitutively expressed in many tissues, e.g., heart, lung, intestine, urogenital system, skin (18), spleen, and tonsils (19). IL-38 is considered to maintain the homeostasis of the microenvironment in these tissues via inhibiting/suppressing inflammatory responses (20). The antiinflammatory role of IL-38 includes the release of IL-38 from apoptotic cells to limit inflammatory macrophage responses (21). An anti-inflammatory role for IL-38 has been demonstrated in a humanized allergic asthma NOD/SCID murine model, showing that IL-38 inhibits proinflammatory cytokines (IL-6, TNF, CCL5, and CXCL10), probably via the classical STAT1, STAT3, p38 MAPK, ERK1/2, and NF- $\mathrm{B}$ pathways (22). IL-38 is analogous to the IL-1ab/receptor agonist and IL-1R1, allowing it to mediate its anti-inflammatory activities. In addition, IL38 plays an important role in maintaining homeostasis by balancing the pro- vs. anti-inflammatory micro-environment (23). Dysregulation of IL-38 can initiate host immunity due to an imbalance of the pro- vs. anti-inflammatory microenvironment, causing inflammatory diseases.

Up-regulated IL-38 expression is detected in inflamed skin (18), the active inflamed tissues of inflammatory bowel disease (24), rheumatoid arthritis (25), psoriatic skin (23), and in druginduced liver injury patients (26), suggesting the induction of an anti-inflammatory role of IL-38 in response to focal inflammation. On the other hand, IL-38 is downregulated in psoriatic skin in response to stimulation by IL-36 $\gamma$, IL-17, and IL-22n (all proinflammatory cytokines) (27). This observation suggests that IL-38 counteracts the biological processes induced by pro-inflammatory cytokines in epithelial and endothelial cells, attenuating the severity of autoimmunity. Exogenous IL38 displays a therapeutic effect in vivo in arthritis in an animal model (28).

\section{COLORECTAL CANCER}

Despite decades of extensive basic and clinical research (29), colorectal cancer (CRC) is still the third leading cause of malignancies (1.93 million cases p.a.) and the second leading cause of cancer-related death $(935,000$ deaths p.a.) worldwide (30). It is still a major challenge to both clinicians and patients to manage $\mathrm{CRC}$, consequently these patients have poor outcomes and 5-year survival rate (31), since a high proportion of CRC patients are diagnosed at a late stage, exhibiting both deep bowel wall invasion and/or distant metastasis.

A key mechanism in tumorigenesis is an immune response to the tumor, illustrated by the effectiveness of immune checkpoint inhibitors (ICIs) acting against, for example, PD-1, PD-L1, and CTLA-4 (32), because of the evidence that blockade of PD1 and PD-L1 is an extremely promising approach in cancer immunotherapy for preventing tumor evasion of host tumor antigen-specific T-cell immunity. At the molecular level, CRC can be divided into mismatch repair/microsatellite instability (MMR/MSI) competent tumors (the majority of CRCs) or MMR/MSI incompetent tumors (approximately $4 \%$ of CRC) (Hirano 2020). The MMR/MSI incompetent form of CRC is considered to be an "immune-hot" form of CRC, characterized by an increased tumor mutational burden, $\mathrm{T}$ cell infiltration, and a substantial anti-tumor immune response within the tumor microenvironment. This form of CRC responds well to ICIs. On the other hand, the majority of CRCs (approximately 96\%) are MMR/MSI competent and tumor growth is primarily driven by increased WNT signaling. These tumors are characterized by an immune-exclusive microenvironment, thought to be due to a low tumor mutational burden. Consequently, MMR/MSI competent tumors respond poorly to ICIs, although current trials are underway to increase the inflammatory response within the tumor microenvironment of this form of CRC, using, for example, radiotherapy to induce inflammation and, thus, improve the response of MMR/MSI competent CRCs to ICIs (33). Host immunity plays a critical role in tumorigenesis, especially in CRC, consistent with reports showing that there is a positive correlation between pro-inflammatory mediators and the severity of CRC (34), especially in CRC associated with MMR/MSI incompetent tumors (35).

Clinical evidence demonstrates that there is substantial macrophage infiltration in cancer tissues, termed tumor associated macrophages (TAM). The role of macrophages during the development of cancer is controversial, it is probably related to the terminal differentiation of macrophages, which can be divided into two subsets, based on their surface markers and functionalities, namely classical activated M1 macrophages and alternatively activated M2 macrophages (36). M1 TAM are believed to typically exert anti-tumor functions, including directly mediated cytotoxicity, e.g., the release of ROS and NO, and antibody-dependent cell-mediated cytotoxicity (ADCC) to kill tumor cells; On the other hand, M2 TAM can promote the occurrence and metastasis of tumor cells, inhibit the $\mathrm{T}$ cell-mediated anti-tumor immune response, promote tumor angiogenesis, and lead to tumor progression (37). Notably, IL-37 has been shown to inhibit the maturation of M2 macrophages (10).

In the current review, we illustrate the relationship between the expression of IL-37 and IL-38, and colorectal adenocarcinoma, especially for its potential clinical implications, because it is the most common colon and 
rectum cancer, derived from epithelial cells of the intestinal mucosa (38).

\section{IL-37 IN COLORECTAL CANCER}

Colorectal cancer exhibits substantially reduced IL-37 at both the mRNA and protein levels, compared to that of non-cancer tissues (39). Importantly, a significant correlation is observed between the level of IL-37 expression and disease-free survival, as well as overall survival (39), suggesting that colonic IL-37 provides protection during the development of CRC. Significantly, the level of colonic IL-37 in CRC tissue is inversely correlated with invasion and differentiation (39). It is known that IL-37 is an anti-inflammatory cytokine (2), and consequently inhibits gastrointestinal inflammation following various pathological stimuli, which ultimately suppresses tumorigenesis in the gut via inhibiting local inflammation (40). However, any long term chronic stimulation could perturb the balance between a proand anti-inflammatory response, resulting in the prolonged persistence of chronic inflammation due to loss of tissue homeostasis (41).

This observation in CRC is partially supported by the findings in gastric cancer, showing that mucosal IL-34 expression is inversely correlated with gastric cancer, including invasion, differentiation, and TNM (42), suggesting that mucosal IL-34 seems to also protect against the development of GC, probably by stimulating the maturation of macrophages via the CSF1 receptor. The comparison of the observations in CRC and gastric cancer appear contradictory, since IL-37 is an antiinflammatory cytokine, whereas IL-34 is a pro-inflammatory cytokine, yet these two cytokines both provide a protective role during the development of cancer within the gastrointestinal system. The potential explanation for such a discrepancy is that there is a substantial difference in the microenvironments between the colon and stomach, although these two are both in the gastrointestinal system, e.g., there is a substantial difference in the bacterial flora ( $>100$-fold) between the colon and stomach, which requires different host mucosal regulatory responses for the maintenance of homeostasis (43). Consequently, there are different pathogenesis pathways for gastric cancer and colon cancer, which may involve the induction of different pathways during tumorigenesis. It has been reported that IL-34 is involved in the M-CSF-mediated pathway to regulate recruitment and/or polarization of TAM, maturing into either M1 and M2 subsets (37), with M-CSF polarizing TAM into the immunosuppressive, cancer-promoting M2 phenotype.

The connection between IL-37 and differentiation has been documented in atherosclerotic development. An inverse correlation has been reported between infiltrating M1 cells and the expression of IL-37 in calcified human aortic valves, suggesting that IL-37 suppresses M1 polarization (44). Paradoxically, IL-37 has been reported to promote M1 polarization $(7,9,10,44)$. Such a discrepancy of IL37 in regulating macrophages between tumorigenesis and atherogenesis may be due to the different systems, i.e., there are a large number of flora in the gut with a great potential to be opportunistic pathogen(s); whereas the cardiovascular system is almost pathogen free. This discrepancy requires further clarification in future.

In addition, it remains to be clarified whether the suppression of IL-37 or IL-34 in CRC and gastric cancer, respectively, results in or from different stimuli, which requires further investigation. A recent study illustrates that exogenous IL-37 inhibits colonic cancer cells and stem cell proliferation and migration in vitro and in a CRC animal model in vivo via the $\beta$-catenin pathway (39). IL-37, belongs to the IL-1 superfamily, and uses the IL-18R and IL-1R8 signaling pathway for regulating its target cells (9), inhibiting dendritic cells. IL-1R8 inactivation is known to be an escape mechanism in CRC (45). However, the precise underlying mechanism involved in such pathways needs further clarification.

Moreover, the right colon is derived from the midgut, while the left side is derived from the hindgut during embryonic development (46). Consequently, there are substantial differences in biological markers, genes and prognosis between left- and right-sided CRC (47). Notably, colonic obstruction from rightsided CRC usually presents at a relatively late stage, compare to that of left-sided CRC (47), due to the more liquid nature of stools and the more capacious nature of the right colon, requiring a more extensive tumor mass to cause obstructive symptoms. However, there is no significant difference in the expression profile of colonic IL-37 between left- and right-sided CRC (39), which may be due to relatively insufficient sensitivity and/or the small sample size, or may be due to a lower protective effect of IL-37 in CRC.

It has been well-established that there is a significant correlation between age and mortality from CRC within a large USA database study (48). To determine whether age is a contributing factor in determining the level of colonic IL-37 expression in CRC patients, a study has been undertaken that compares IL-37 expression between old ( $>65$ year) and young $(<65$ year) CRC patients. Interestingly, no significant difference is observed in colonic IL-37 levels between old ( $>65$ year) and young ( $<65$ year) CRC patients, suggesting that age is not a contributory factor in determining the level of IL-37 expression. This discrepancy may be due to the majority of the CRC patients studied being close to 65 years, which would reduce the power of age in the study, and/or there may have been an insufficient sample size, particularly from a single medical center (39). Thus, a larger sample size and the use of multiple centers for a study, with different racial background, should be undertaken in a future study. In addition, there is no significant difference in IL-37 levels between males and females, most likely mainly due to most females within the study being post-menopausal, which negates the known benefit of estrogen in reducing the incidence of CRC in women of fertile age (49).

Another research team has shown that IL-37 is localized in the cytoplasm of colonic epithelial cells, but colonic IL-37 expression from CRC tissues is substantially reduced compared to that of non-CRC colonic epithelial cells (50). Colonic IL37 expression is inversely correlated with the depth of CRC invasion, which is consistent with CRC progression (50). In addition, IL-37 expression is closely related to overall disease-free status and overall survival. Surprisingly, there is no significant 
correlation between colonic IL-37 expression and differentiation of CRC (50). The data obtained in this study may be related to the sensitivity and specificity of the quantification used in the study, i.e., the IL-37 immunohistochemistry utilized a semi-quantitative, semi-subjective methodology using simple visual inspection, which might compromise objective evaluation. Ideally, an immunohistochemical staining quantification using an automated computerized system, e.g., ImagePro Plus, should be used for more objective and accurate evaluation (42).

The role of tumor associated neutrophils (TAN) is a rather novel concept, suggesting that TANs can promote development of tumors via enhancing inflammation and subsequent angiogenesis and extracellular matrix remodeling and metastasis (51). It has been demonstrated that a combination of IL-37 and TANs seems to be a reliable prognostic biomarker for CRC, using multivariate analysis (50). The finding suggests that IL-37 also reduces inflammation in the microenvironment by inhibiting recruitment of neutrophils, which is well-known as an acute and/or chronic source of inflammation (50).

The protective role of IL-37 in tumors has been demonstrated in a number of cancers, e.g. in human hepatocellular carcinoma (52), perhaps via inhibiting M2 macrophages (10), in lung cancer (53) via inhibiting angiogenesis, and in an animal model (54). Furthermore, a high level of IL-37 has been shown to correlate with increased $\mathrm{CD}^{2} \mathrm{a}^{+}$dendritic cell infiltration, and correlates well with the overall survival rate in hepatocellular carcinoma (8). The consequence of increased dendritic cells in antitumor immunity perhaps involves enhanced professional antigen presentation with subsequent increased differential polarization of macrophages (8). This could explain the differential role of macrophages during the development of malignancies, which may exhibit different mechanisms of carcinogenesis or microenvironments.

\section{IL-38 AND CRC}

IL-38 is constitutively expressed in very low amounts in many tissues and has an anti-inflammatory role (20) for maintaining homeostasis within the micro-environment. The anti-inflammatory role of IL-38 includes the release of IL-38 from apoptotic cells to limit inflammatory macrophage responses (21). Thus, any disturbance of IL-38 can cause maladaptive clinical responses. From a functionality point of view, IL-38 displaces inflammatory signaling, analogous to the IL-1ab/receptor agonist and IL-1R1, as an anti-inflammatory mediator.

Chen et al. found that colonic IL-38 production in CRC is mainly located in the cytoplasm of epithelial cells in non-CRC colonic tissue, but is substantially down-regulated within CRC colonic tissue compared to that of matched non-CRC colonic tissue (55), suggesting that IL-38 is involved in the development of CRC. This is supported by the finding that an inverse correlation is also detected between colonic IL-38 production and the extent of differentiation of CRC, suggesting a protective role of colonic IL-38 to maintain normal homeostasis of the intestinal mucosa within the micro-environment. The protective role of
IL-38 in CRC is in line with other studies, showing that IL-38 provides protection against gestational diabetes mellitus-induced inflammation within the placenta (56), and in the context of the demonstration of a close correlation between inflammatory bowel disease and the development of CRC (57).

As stated above, there are physiological and anatomic differences between the right and left sides of the colon (46), arising from embryonic development, which contributes to different clinical symptoms for left- and right-sided CRC patients, resulting in a later diagnosis of right-sided colon cancers (47). Colonic IL-38 expression has been shown to be nearly half the level in right-sided CRC compared to that of the left side (55). The precise underlying mechanism is unclear, but it may be related to the differentiation and/or extent of invasion of CRC, correlating to clinical symptoms during the development of CRC. In addition, defective MMR/MSI positive CRC is more frequent in right-sided CRC and is associated with a higher level of inflammation within the tumor microenvironment. Thus, low levels of IL-38 may be a complex reflection of this higher level of inflammation. The relationship between MSI and IL-38 expression has not yet been investigated.

These data also correlate with the finding that reduced colonic IL-38 is detected in larger CRCs with a tumor size $>5 \mathrm{~cm}$, compared to that of $\leq 5 \mathrm{~cm}$ (55). Notably, right-sided CRC is frequently diagnosed at a later stage, with the tumor size being larger, due to the capacious nature of the proximal colon (47). It is well known that the tumor size and depth of invasion (58) correlates with prognosis and overall survival rate of primary CRC patients (59).

As stated above, age is an important factor during the development of CRC (48), however, no significant difference of colonic IL-38 is detected between young and old (cut-off at 70 years) CRC patients. This may be due to the majority of the CRC patients within the study being within a relatively old group, close to 65 years, which may dilute the power of the statistics, and/or the sample size being relatively small and from a single center (55). Similarly, no significant difference in CRC IL-38 expression is observed between males and females, which is consistent with similar findings for colonic IL-37 expression in CRC tissues between males and females $(39,50)$, and is probably due to the majority of these CRC female patients being postmenopausal, consequently having already lost the protective power of estrogen (49).

Although the precise underlying mechanism involved in the reduction of colonic IL-38 during the development of CRC is unclear, it is speculated that compromised intestinal mucosal immunity might contribute to the disturbance of the balance between pro- and anti-inflammatory cytokines in the colon (27) within the susceptible cohort (60). Consequently, the resultant dysregulated inflammation within the intestinal mucosal immunity of these individuals (61) is eventually conducive to the development of CRC $(34,62)$. Furthermore, the finding that IL-38 is also inversely correlated with TNM classification in CRC (55), suggests that reduced local and, probably, circulating IL-38 might promote malignant cell shortdistance invasion or long-distance metastasis. This is further supported by the findings that there is a close correlation between 
chronic inflammation and the development of gastrointestinal cancer (63).

In contrast, a correlation has been reported between high IL-38 expression and poor differentiation of lung adenocarcinoma (64). Moreover, within this lung cancer cohort, high IL-38 expression also positively correlated with high TNM and with a shorter disease-free survival, but only in PD-L1 negative tumors where $\mathrm{T}$ cell activity is presumably not suppressed. These data suggest that IL38 promotes the development of lung carcinoma, with an inverse correlation with $\mathrm{PD}-1 / \mathrm{PD}-\mathrm{L} 1$ expression, rather than inhibits the development of lung cancer. The precise underlying mechanism/s explaining the apparently contradictory involvement of IL-38 in CRC compared to lung cancer is still unclear. We speculate that the discrepancy in IL-38 expression in CRC vs. non-small cell carcinoma of the lung may be due to different immune system responses within the two tissues, which experience quite different microflora loads and/or microenvironments, with the differing stimuli tipping the different host responses in opposite directions, which requires further clarification.

It is well-known that the IL-36 isoforms are pro-inflammatory cytokines, which contribute to activation and proliferation of leucocytes, including macrophages, dendritic cells, and lymphocytes (65). It has been demonstrated that there are differential roles for IL-36 $\alpha, \beta$, and $\gamma$ in CRC (66), when the level of expression of these interleukins is evaluated for their capacity to predict 5-year survival among CRC patients. Considering that the pathophysiological function of IL-38 is to bind via the IL36 receptor to block IL-36 signaling $(20,67)$, these data are consistent with the observed correlation between high expression of IL-38 and improved 5-year survival in CRC (55). The direct interaction between IL-38 and IL-36 during the development of CRC remains to be explored. Our preliminary observations have shown the induction of larger and more numerous CRCs

\section{REFERENCES}

1. Dinarello C, Arend W, Sims J, Smith D, Blumberg H, O’Neill L, et al. IL-1 family nomenclature. Nat Immunol. (2010). 11:973. doi: 10.1038/ni1110-973

2. Nold MF, Nold-Petry CA, Zepp JA, Palmer BE, Bufler P, Dinarello CA. IL37 is a fundamental inhibitor of innate immunity. Nat Immunol. (2010) 11:1014-22. doi: 10.1038/ni.1944

3. Boraschi D, Lucchesi D, Hainzl S, Leitner M, Maier E, Mangelberger D, et al. IL-37: a new anti-inflammatory cytokine of the IL-1 family. Eur Cytokine Netw. (2011) 22:127-47. doi: 10.1684/ecn.2011.0288

4. Pan G, Risser P, Mao W, Baldwin DT, Zhong AW, Filvaroff E, et al. IL-1H, an interleukin 1-related protein that binds IL-18 receptor/IL-1Rrp. Cytokine. (2001) 13:1-7. doi: 10.1006/cyto.2000.0799

5. Smith DE, Renshaw BR, Ketchem RR, Kubin M, Garka KE, Sims JE. Four new members expand the interleukin-1 superfamily. J Biol Chem. (2000) 275:1169-75. doi: 10.1074/jbc.275.2.1169

6. Luo Y, Cai X, Liu S, Wang S, Nold-Petry CA, Nold MF, et al. Suppression of antigen-specific adaptive immunity by IL-37 via induction of tolerogenic dendritic cells. Proc Nat Acad Sci USA. (2014) 111:1517883. doi: 10.1073/pnas.1416714111

7. Cavalli G, Dinarello CA. Suppression of inflammation and acquired immunity by IL-37. Immunol Rev. (2018) 281:179-90. doi: 10.1111/imr.12605 in IL-38 GKO animals compared to the wild-type, supporting speculation that IL-38 plays a critical protective role during the development of CRC, perhaps via regulating host immunity. Although the counteractive role between IL-38 (55) and IL36s (66) in the development of CRC remains to be confirmed, considering the known anti-inflammatory role of IL-38 and proinflammatory role of the IL-36 isoforms, this mechanism likely contributes to the dysregulated intestinal immunity that occurs during CRC pathogenesis.

In conclusion, this mini review makes the case that there are some similarities between IL-37 and IL-38 in inhibiting tumorigenesis of CRC, yet these two interleukins use different signaling pathways in mediating and/or interacting with cancer cells in the tumor microenvironment. IL-37 and IL-38 are reliable prediction factors for determining the prognosis of CRC, and constitute strong and reliable predictors of survival postsurgery. There is room for improvement in these supporting data, including the extension of the study to different regions/countries evaluating different races, determining the effects of multi-drug resistance, and clarifying expression levels in morphological and molecular subsets of CRC. It would be useful to determine the kinetics of circulating IL-38 and its relationship with drug resistance/targeted therapy. The measurement of colonic IL-38 at both the molecular and cellular level is required to explore the contribution of IL-38 pathways during the development of CRC. Furthermore, these data also suggest that IL-37 and IL-38 may serve as therapeutic targets in the post-surgery management of CRC patients, particularly in the development of personalized precision medicine.

\section{AUTHOR CONTRIBUTIONS}

JD, ZH, XC, JF, DH, BH, XL, and SB conceptualized the idea and contributed to writing the article. All authors contributed to the article and approved the submitted version.
8. Liu Y, Zhao JJ, Zhou ZQ, Pan QZ, Zhu Q, Tang Y, et al. IL-37 induces anti-tumor immunity by indirectly promoting dendritic cell recruitment and activation in hepatocellular carcinoma. Cancer Manag Res. (2019) 11:6691702. doi: 10.2147/CMAR.S200627

9. Liu T, Liu J, Lin Y, Que B, Chang C, Zhang J, et al. IL-37 inhibits the maturation of dendritic cells through the IL-1R8-TLR4-NF$\kappa \mathrm{B}$ pathway. Biochim Biophys Acta Mol Cell Biol Lipids. (2019) 1864:1338-49. doi: 10.1016/j.bbalip.2019.05.009

10. Zhang Z, Zhang J, He P, Han J, Sun C. Interleukin-37 suppresses hepatocellular carcinoma growth through inhibiting M2 polarization of tumor-associated macrophages. Mol Immunol. (2020) 122:1320. doi: 10.1016/j.molimm.2020.03.012

11. Ye L, Jiang B, Deng J, Du J, Xiong W, Guan Y, et al. IL-37 Alleviates rheumatoid arthritis by suppressing IL-17 and IL-17-triggering cytokine production and limiting Th17 cell proliferation. J Immunol. (2015) 194:51109. doi: 10.4049/jimmunol.1401810

12. Li Y, Wang Y, Liu Y, Wang Y, Zuo X, Li Y, et al. The possible role of the novel cytokines il-35 and il-37 in inflammatory bowel disease. Mediators Inflamm. (2014) 2014:136329. doi: 10.1155/2014/136329

13. Yu Z, Liu J, Zhang R, Huang X, Sun T, Wu Y, et al. IL-37 and 38 signalling in gestational diabetes. J Reprod Immunol. (2017) 124:814. doi: 10.1016/j.jri.2017.09.011 
14. Teng MWL, Bowman EP, McElwee JJ, Smyth MJ, Casanova J-L, Cooper $\mathrm{AM}$, et al. IL-12 and IL-23 cytokines: from discovery to targeted therapies for immune-mediated inflammatory diseases. Nat Med. (2015) 21:71929. doi: 10.1038/nm.3895

15. Law CC, Puranik R, Fan J, Fei J, Hambly BD, Bao S. Clinical implications of IL-32, IL-34 and IL-37 in atherosclerosis: speculative role in cardiovascular manifestations of COVID-19. Front Cardiovasc Med. (2021) 8:630767. doi: 10.3389/fcvm.2021.630767

16. Liu J, Lin J, He S, Wu C, Wang B, Liu J, et al. Transgenic overexpression of IL-37 protects against atherosclerosis and strengthens plaque stability. Cell Physiol Biochem. (2018) 45:1034-50. doi: 10.1159/000487344

17. Mulero JJ, Pace AM, Nelken ST, Loeb DB, Correa TR, Drmanac R, et al. IL1HY1: a novel interleukin-1 receptor antagonist gene. Biochem Biophys Res Commun. (1999) 263:702-6. doi: 10.1006/bbrc.1999.1440

18. Debets R, Timans JC, Homey B, Zurawski S, Sana TR, Lo S, et al. Two novel IL1 family members, IL-1 delta and IL-1 epsilon, function as an antagonist and agonist of NF-kappa B activation through the orphan IL-1 receptor-related protein 2. J Immunol. (2001) 167:1440-6. doi: 10.4049/jimmunol.167.3.1440

19. Lin H, Ho AS, Haley-Vicente D, Zhang J, Bernal-Fussell J, Pace AM, et al. Cloning and characterization of IL-1HY2, a novel interleukin-1 family member. J Biol Chem. (2001) 276:20597-602. doi: 10.1074/jbc.M010095200

20. Bao S, Hu R, Hambly BD. IL-34, IL-36 and IL-38 in colorectal cancerkey immunoregulators of carcinogenesis. Biophys Rev. (2020) 12:92530. doi: 10.1007/s12551-020-00726-0

21. Mora J, Schlemmer A, Wittig I, Richter F, Putyrski M, Frank $\mathrm{AC}$, et al. Interleukin-38 is released from apoptotic cells to limit inflammatory macrophage responses. J Mol Cell Biol. (2016) 8:426-38. doi: 10.1093/jmcb/mjw006

22. Sun X, Hou T, Cheung E, Iu TN, Tam VW, Chu IM, et al. Anti-inflammatory mechanisms of the novel cytokine interleukin-38 in allergic asthma. Cell Mol Immunol. (2020) 17:631-46. doi: 10.1038/s41423-019-0300-7

23. Mercurio L, Morelli M, Scarponi C, Eisenmesser EZ, Doti N, Pagnanelli G, et al. IL-38 has an anti-inflammatory action in psoriasis and its expression correlates with disease severity and therapeutic response to anti-IL-17A treatment. Cell Death Dis. (2018) 9:1104. doi: 10.1038/s41419-018-1143-3

24. Fonseca-Camarillo G, Furuzawa-Carballeda J, Iturriaga-Goyon E, YamamotoFurusho JK. Differential expression of IL-36 family members and IL-38 by immune and nonimmune cells in patients with active inflammatory bowel disease. Biomed Res Int. (2018) 2018:5140691. doi: 10.1155/2018/5140691

25. Takenaka S-i, Kaieda S, Kawayama T, Matsuoka M, Kaku Y, Kinoshita T, et al. IL-38: a new factor in rheumatoid arthritis. Biochem Biophys Rep. (2015) 4:386-91. doi: 10.1016/j.bbrep.2015.10.015

26. Kumar N, Surani S, Udeani G, Mathew S, John S, Sajan S, et al. Drug-induced liver injury and prospect of cytokine based therapy. A focus on IL-2 based therapies. Life Sci. (2021) 278:119544. doi: 10.1016/j.lfs.2021.119544

27. van de Veerdonk FL, de Graaf DM, Joosten LAB, Dinarello CA. Biology of IL-38 and its role in disease. Immunol Rev. (2018) 281:1916. doi: 10.1111/imr.12612

28. Boutet M-A, Najm A, Bart G, Brion R, Touchais S, Trichet V, et al. IL-38 overexpression induces anti-inflammatory effects in mice arthritis models and in human macrophages in vitro. Ann Rheum Dis. (2017) 76:1304. doi: 10.1136/annrheumdis-2016-210630

29. Keum N, Giovannucci E. Global burden of colorectal cancer: emerging trends, risk factors and prevention strategies. Nat Rev Gastroenterol Hepatol. (2019) 16:713-32. doi: 10.1038/s41575-019-0189-8

30. WHO. Cancer. (2021).

31. Society AC. Survival Rates for Colorectal Cancer 2021. Available online at: https://www.cancer.org/cancer/colon-rectal-cancer/detection-diagnosisstaging/survival-rates.html (accessed January 29, 2021).

32. Gong J, Chehrazi-Raffle A, Reddi S, Salgia R. Development of PD-1 and PDL1 inhibitors as a form of cancer immunotherapy: a comprehensive review of registration trials and future considerations. J Immunother Cancer. (2018) 6:8. doi: 10.1186/s40425-018-0316-z

33. Weichselbaum RR, Liang $H$, Deng L, Fu YX. Radiotherapy and immunotherapy: a beneficial liaison? Nat Rev Clin Oncol. (2017) 14:365-79. doi: 10.1038/nrclinonc.2016.211

34. Coussens LM, Werb Z. Inflammation and cancer. Nature. (2002) 420:8607. doi: $10.1038 /$ nature 01322
35. Waldman AD, Fritz JM, Lenardo MJ. A guide to cancer immunotherapy: from T cell basic science to clinical practice. Nat Rev Immunol. (2020) 20:651-68. doi: 10.1038/s41577-020-0306-5

36. Martinez FO, Gordon S. The M1 and M2 paradigm of macrophage activation: time for reassessment. F1000Prime Rep. (2014). 6:13. doi: 10.12703/P6-13

37. Pan Y, Yu Y, Wang X, Zhang T. Tumor-associated macrophages in tumor immunity. Front Immunol. (2020) 11:583084. doi: 10.3389/fimmu.2020.583084

38. Fleming $M$, Ravula S, Tatishchev SF, Wang HL. Colorectal carcinoma: pathologic aspects. J Gastrointest Oncol. (2012) 3:153-73. doi: 10.3978/j.issn.2078-6891.2012.030

39. Yan X, Zhao J, Zhang R. Interleukin-37 mediates the antitumor activity in colon cancer through beta-catenin suppression. Oncotarget. (2017) 8:4906475. doi: 10.18632/oncotarget.17093

40. Jemal A, Siegel R, Xu J, Ward E. Cancer statistics, 2010. CA Cancer J Clin. (2010) 60:277-300. doi: 10.3322/caac.20073

41. Medzhitov R. Origin and physiological roles of inflammation. Nature. (2008) 454:428-35. doi: 10.1038/nature07201

42. Liu Q, Zhang Y, Zhang J, Tao K, Hambly BD, Bao S. Inverse correlation between Interleukin-34 and gastric cancer, a potential biomarker for prognosis. Cell Biosci. (2020) 10:94. doi: 10.1186/s13578-020-00454-8

43. Klatt EC. The gastrointestinal tract. In: Kumar V, Abbas A, Aster JC, editors. Robbins \& Cotran Pathologic Basis of Disease. Philadelphia, PA: SaundersElsevier (2015). p. 177-223.

44. Zhou P, Li Q, Su S, Dong W, Zong S, Ma Q, et al. Interleukin 37 suppresses M1 macrophage polarization through inhibition of the Notch1 and nuclear factor kappa b pathways. Front Cell Dev Biol. (2020) 8:56. doi: 10.3389/fcell.2020.00056

45. Molgora M, Barajon I, Mantovani A, Garlanda C. Regulatory role of IL-1R8 in immunity and disease. Front Immunol. (2016) 7:149. doi: 10.3389/fimmu.2016.00149

46. Kostouros A, Koliarakis I, Natsis K, Spandidos DA, Tsatsakis A, Tsiaoussis J. Large intestine embryogenesis: molecular pathways and related disorders (Review). Int J Mol Med. (2020) 46:27-57. doi: 10.3892/ijmm.2020.4583

47. Venook AP. Right-sided vs left-sided colorectal cancer. Clin Adv Hematol Oncol. (2017) 15:22-4.

48. Siegel RL, Miller KD, Goding Sauer A, Fedewa SA, Butterly LF, Anderson JC, et al. Colorectal cancer statistics, 2020. CA Cancer J Clin. (2020) 70:14564. doi: $10.3322 /$ caac. 21601

49. Lin JH, Giovannucci E. Sex hormones and colorectal cancer: what have we learned so far? J Natl Cancer Inst. (2010) 102:1746-7. doi: 10.1093/jnci/djq444

50. Zhu B, Luo J, Jiang Y, Yu L, Liu M, Fu J. Prognostic significance of nomograms integrating IL-37 expression, neutrophil level, and MMR status in patients with colorectal cancer. Cancer Med. (2018) 7:368294. doi: 10.1002/cam4.1663

51. Jaillon S, Ponzetta A, Di Mitri D, Santoni A, Bonecchi R, Mantovani A. Neutrophil diversity and plasticity in tumour progression and therapy. Nat Rev Cancer. (2020) 20:485-503. doi: 10.1038/s41568-020-0281-y

52. Zhao JJ, Pan QZ, Pan K, Weng DS, Wang QJ Li JJ, et al. Interleukin-37 mediates the antitumor activity in hepatocellular carcinoma: role for CD57+ NK cells. Sci Rep. (2014) 4:5177. doi: 10.1038/srep05177

53. Ge G, Wang A, Yang J, Chen Y, Yang J, Li Y, et al. Interleukin-37 suppresses tumor growth through inhibition of angiogenesis in non-small cell lung cancer. J Exp Clin Cancer Res. (2016) 35:13. doi: 10.1186/s13046-0160293-3

54. Gao W, Kumar S, Lotze MT, Hanning C, Robbins PD, Gambotto A. Innate immunity mediated by the cytokine IL-1 homologue 4 (IL-1H4/IL-1F7) induces IL-12-dependent adaptive and profound antitumor immunity. $J$ Immunol. (2003) 170:107-13. doi: 10.4049/jimmunol.170.1.107

55. Chen F, Zhang F, Tan Z, Hambly BD, Bao S, Tao K. Interleukin-38 in colorectal cancer: a potential role in precision medicine. Cancer Immunol Immunother. (2020) 69:69-79. doi: 10.1007/s00262-019-02440-7

56. Yu H, Liu Y, Xie W, Xie Q, Liu Q, Cheng L. IL-38 alleviates the inflammatory response and the degeneration of nucleus pulposus cells via inhibition of the NF-kappaB signaling pathway in vitro. Int Immunopharmacol. (2020) 85:106592. doi: 10.1016/j.intimp.2020.106592

57. Rogler G. Chronic ulcerative colitis and colorectal cancer. Cancer Lett. (2014) 345:235-41. doi: 10.1016/j.canlet.2013.07.032 
58. Kato T, Alonso S, Muto Y, Perucho M, Rikiyama T. Tumor size is an independent risk predictor for metachronous colorectal cancer. Oncotarget. (2016) 7:17896-904. doi: 10.18632/oncotarget.7555

59. Saha S, Kanaan MN, Shaik M, Abadeer B, Korant A, Krishnamoorthy M, et al. Tumor size as a prognostic factor for patients with colon cancer undergoing sentinel lymph node mapping and conventional surgery. J Clin Oncol. (2013). 31(4_suppl):546. doi: 10.1200/jco.2013.31.4_suppl.546

60. Ummarino D. Experimental arthritis: IL-38 promotes anti-inflammatory effects. Nat Rev Rheumatol. (2017) 13:260. doi: 10.1038/nrrheum.2017.55

61. Elinav E, Nowarski R, Thaiss CA, Hu B, Jin C, Flavell RA. Inflammationinduced cancer: crosstalk between tumours, immune cells and microorganisms. Nat Rev Cancer. (2013) 13:759-71. doi: 10.1038/nrc3611

62. Grivennikov SI. Inflammation and colorectal cancer: colitisassociated neoplasia. Semin Immunopathol. (2013) 35:22944. doi: 10.1007/s00281-012-0352-6

63. Ullman TA, Itzkowitz SH. Intestinal inflammation and cancer. Gastroenterology. (2011) 140:1807-16. doi: 10.1053/j.gastro.2011.01.057

64. Takada K, Okamoto T, Tominaga M, Teraishi K, Akamine T, Takamori S, et al. Clinical implications of the novel cytokine IL-38 expressed in lung adenocarcinoma: possible association with PD-L1 expression. PLoS ONE. (2017) 12:e0181598. doi: 10.1371/journal.pone.0181598

65. Vigne S, Palmer G, Lamacchia C, Martin P, Talabot-Ayer D, Rodriguez E, et al. IL-36R ligands are potent regulators of dendritic and T cells. Blood. (2011) 118:5813-23. doi: 10.1182/blood-2011-05-356873
66. Chen F, Qu M, Zhang F, Tan Z, Xia Q, Hambly BD, et al. IL-36s in the colorectal cancer: is interleukin 36 good or bad for the development of colorectal cancer? BMC Cancer. (2020) 20:92. doi: 10.1186/s12885-020-6587-z

67. Catalan-Dibene J, McIntyre LL, Zlotnik A. Interleukin 30 to Interleukin 40. J Interferon Cytokine Res. (2018) 38:423-39. doi: 10.1089/jir.2018.0089

Conflict of Interest: The authors declare that the research was conducted in the absence of any commercial or financial relationships that could be construed as a potential conflict of interest.

Publisher's Note: All claims expressed in this article are solely those of the authors and do not necessarily represent those of their affiliated organizations, or those of the publisher, the editors and the reviewers. Any product that may be evaluated in this article, or claim that may be made by its manufacturer, is not guaranteed or endorsed by the publisher.

Copyright (C) 2022 Dang, He, Cui, Fan, Hambly, Hambly, Li and Bao. This is an open-access article distributed under the terms of the Creative Commons Attribution License (CC BY). The use, distribution or reproduction in other forums is permitted, provided the original author(s) and the copyright owner(s) are credited and that the original publication in this journal is cited, in accordance with accepted academic practice. No use, distribution or reproduction is permitted which does not comply with these terms. 